Article

\title{
Synthesis and Application of
} $N$-methylphthalimidylazo Disperse Dyes to Cellulose Diacetate for High Wash Fastness

\author{
Sanghyun Yoon ${ }^{1}$, Hyunjung Kim ${ }^{1}$, Eunkyo Lee ${ }^{1}$, Nahyun $\mathrm{Oh}^{1}{ }^{1}$, Sangcheon Kim ${ }^{1}$, \\ Kyung Hwa Hong ${ }^{2}$ and Joonseok Koh 1,3,*(D) \\ 1 Department of Organic and Nano System Engineering, Konkuk University, 120 Neungdong-ro, \\ Gwangjin-gu, Seoul 05029, Korea; sucre92@naver.com (S.Y.); bona111491@naver.com (H.K.); \\ dldmsry1995@naver.com (E.L.); gina444@naver.com (N.O.); poi248@naver.com (S.K.) \\ 2 Department of Fashion Design and Merchandising, Kongju National University, Chungnam 32588, Korea; \\ hkh713@kongju.ac.kr \\ 3 Division of Chemical Engineering, Konkuk University, 120 Neungdong-ro, Gwangjin-gu, Seoul 05029, Korea \\ * Correspondence: ccdjko@konkuk.ac.kr
}

Received: 21 September 2020; Accepted: 28 October 2020; Published: 2 November 2020

\begin{abstract}
Cellulose diacetate fibers were prepared from cellulosic biomass with high $\alpha$-cellulose contents such as purified cotton linters and wood pulps. Cellulose diacetate fibers are sensitive to alkaline solution, which causes hydrolysis of the acetate ester to hydroxyl groups, especially at high temperatures. Thus, the low alkali-resistance of cellulose acetate fibers makes it difficult to achieve high wash fastness by restricting the application of intense after-treatment, such as reduction clearing. A series of $\mathrm{N}$-methylphthalimide-based high-washable azo disperse dyes were synthesized and their dyeing and fastness properties on cellulose diacetate fabrics were investigated. From the overall results obtained in this study, $\mathrm{N}$-methylphthalimidylazo disperse dyes are expected to be a desirable alternative to high value-added dyes that can be used for high color fastness dyeing of cellulose diacetate with a minimal discharge of wastewater during washing process.
\end{abstract}

Keywords: phthalimide; disperse dyes; cellulose diacetate; build-up property; color fastness property; wash-off property

\section{Introduction}

Cellulose and its derivatives are attracting increasing attention as renewable, economical, and abundant resources to reduce dependency on fossil resources [1-3]. Cellulose acetate has been recognized as by far the most important derivative of cellulose because of its versatile industrial and commercial applications [4,5]. Acetate fibers are synthetic cellulosic fibers manufactured by treating cellulosic biomass, such as pure wood pulp or cotton linters, with a mixture of acetic anhydride and acetic acid at a low temperature in the presence of a catalyst such as sulfuric acid, perchloric acid, zinc chloride, or similar salts [6]. The cellulose hydroxyl groups are esterified by the acetic anhydride to form cellulose acetates-the fiber forming components. Cellulose acetate is majorly classified into two types: cellulose triacetate and cellulose diacetate, with acetylation degrees of 3.00 and 2.48 , respectively (Figure 1) [7,8].

Cellulose diacetate fibers are widely used in the apparel industry because of their superior properties, such as silk-like softness, luster, comfort, and good draping quality. However, dyes with satisfactory properties-non-toxicity, environmentally friendliness, and high color fastness, have been a major impediment in their use in the textile industry. Due to their hydrophobic nature, efficient dyeing requires the use of dyes with limited water solubility; disperse dyes are sparingly soluble in 
water and are almost exclusively used for cellulose diacetate fibers. The insolubility of disperse dyes enables them to transfer from water to the surface of hydrophobic fibers, and the application of heat to the dye liquor accelerates the disperse dye's diffusion into the interior of the fiber above its glass transition temperature (Figure 2) [8].

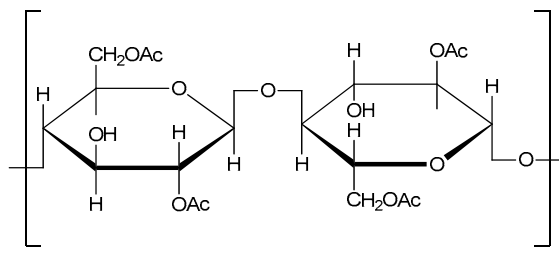

(a)

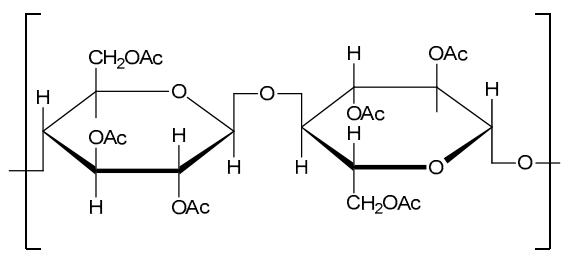

(b)

Figure 1. Chemical structures of acetate fibers: (a) Cellulose diacetate. (b) Cellulose triacetate $\left(\mathrm{Ac}=\mathrm{CH}_{3} \mathrm{CO}\right)$.

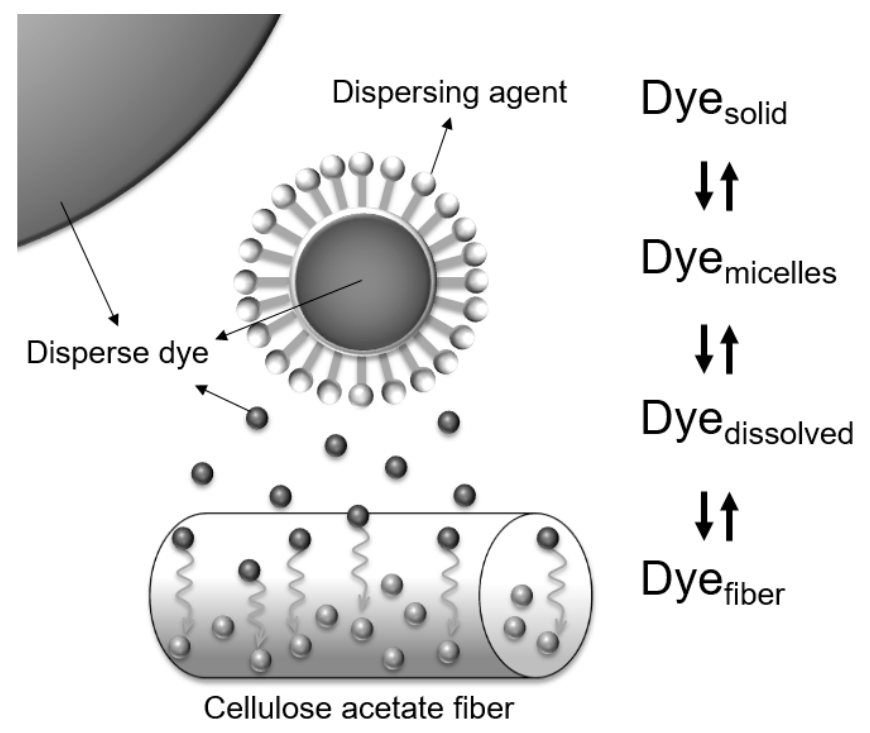

Figure 2. Disperse dyeing mechanism of cellulose acetate fiber.

While the development of disperse dyes was a major breakthrough, their use for cellulose diacetate fibers is faced with numerous challenges and they are consequently limited as compared to other fabrics. To achieve optimal color fastness during disperse dyeing of polyester fiber, excess dye on the fiber surface is cleared by reductive treatment in a strong reduction bath made up of a toxic reducing agent (i.e., sodium hydrosulfite) and a strong alkali (i.e., sodium hydroxide) [9]. However, this is not achievable with cellulose diacetate. During reduction-clearing, the acetyl groups are hydrolyzed, leading to the deterioration of their characteristic properties (Scheme 1). Alternatively, the dyed acetate fabrics are aftertreated with soaping agents at a relatively low temperature. Although this method retains their unique characteristics, the resultant color fastness properties are not satisfactory [8]. In this case, fastness can be improved by washing several times using large amounts of wash water, which consequently increases the wastewater load in the dyehouse effluent. 


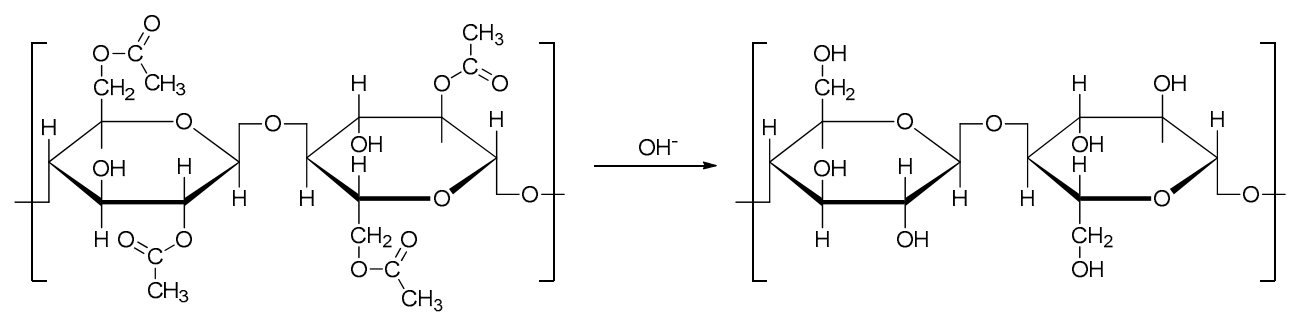

Scheme 1. Alkali-hydrolysis of cellulose diacetate fibers.

In our previous study, we demonstrated phthalimidylazo-based alkali-clearable disperse dyes as a solution to environmental problems in the polyester dyeing industry, which subsequently eliminated the need for a toxic reducing agent and a strong alkali, thereby reducing the cost of wastewater treatment [10]. Azo disperse dyes incorporating a phthalimide group hydrolyze to dyes containing a water-solubilizing diester group under relatively mild alkaline to neutral conditions without causing cleavage of the azo bond and are easily washed-off (Scheme 2). The hydrolysis mechanism of phthalimidylazo disperse dyes was verified through analysis of the dye hydrolysis under mild alkali conditions by FT-IR (Fourier-transform infrared), mass, and UV- Vis (Ultraviolet-visible) spectrophotometry. HPLC (High performance liquid chromatography) analysis showed that mild alkaline treatment $\left(\mathrm{Na}_{2} \mathrm{CO}_{3} 1.0 \mathrm{~g} / \mathrm{L}\right)$ of phthalimidylazo disperse dyes at $80^{\circ} \mathrm{C}$ for 20 min resulted in more than $95 \%$ hydrolysis of parent dyes and the overall color fastness of dyed polyester was in the range of $4-5$ to $5[10,11]$. However, the phthalimidylazo disperse dyes showed excellent stability under the conventional high temperature disperse dyeing condition. i.e., less than $3 \%$ hydrolysis during dyeing at $130{ }^{\circ} \mathrm{C}$ for $60 \mathrm{~min}$ ( $\mathrm{pH}$ 4.5). Therefore, the use of highly washable phthalimidylazo disperse dyes is expected to ensure excellent stability during dyeing and improved color fastness properties of cellulose diacetate fibers with a mild aftertreatment, such as soaping treatment, without compromising their unique characteristics.<smiles>[R]c1cc(N([R])[R])c([R])cc1N=Nc1ccc2c(c1)C(=O)N([R])C2=O</smiles><smiles>[R]c1cc(N([R])[R])c([R])cc1N=Nc1ccc(C(=O)O[Na])c(C(=O)ONC(N)=O)c1</smiles>

Scheme 2. Alkali-hydrolysis of phthalimidylazo disperse dyes under milk alkaline condition $\left(\mathrm{Na}_{2} \mathrm{CO}_{3}\right.$ $1.0 \mathrm{~g} / \mathrm{L}, 80^{\circ} \mathrm{C}$ for $20 \mathrm{~min} ; \mathrm{R}^{1}=$ alkyl, phenyl; $\mathrm{R}^{2}, \mathrm{R}^{3}=\mathrm{H}, \mathrm{CH}_{3}, \mathrm{OCH}_{3}, \mathrm{NHCOCH}_{3} ; \mathrm{R}^{4}, \mathrm{R}^{5}=$ substituted alkyls).

In the present study, $N$-methylphthalimide-based highly washable azo disperse dyes were synthesized and their dyeing and fastness properties on cellulose acetate fabrics were compared with those of typical 4-aminoazobenzene disperse dyes. Specifically, in order to increase the affinity of phthalimidylazo disperse dyes for cellulose diacetate fibers having a relatively less hydrophobic nature than polyester fibers, hydrophilic substituents were introduced into the coupling component of the dyes.

\section{Materials and Methods}

\subsection{Materials and Reagents}

The chemicals used in the synthesis of disperse dyes were $N$-methyl-4-aminophthalimide, $N, N$ -diethylaniline, 2-(N-ethylanilino)ethanol, $N$-phenyldiethanolamine, $N, N$-diethyl-m-toluidine, 2-( $N$ -ethyl-m-toluidino)ethanol, m-tolyldiethanolamine, $N, N$-dimethylformamide, dimethyl sulfoxide-d6 
(DMSO-d6), sodium nitrite, sodium acetate, and methanol. Cellulose diacetate fabrics $\left(70 \pm 5 \mathrm{~g} / \mathrm{m}^{2}\right.$; plain weave, warp, and weft each 75 denier) were used for disperse dyeing. All chemicals used for the synthesis and dyeing were of laboratory reagent grade. Dispersing agent (Diwatex, Borregaard Lignotech, anionic, Sarpsborg, Norway) and wetting agents (Sandozin NIE, Clariant, nonionic, Muttenz, Switzerland) were used for milling. Lyocol RDN liquid (Clariant, anionic, Muttenz, Switzerland) was used as a dispersing agent in dyeing experiments. Inkanol AZ-100 (Poong Yeong Chem, nonion, Gyeonggi-do, Korea) was used as a soaping agent in aftertreatment.

\subsection{Instrumentation}

The IR spectra were obtained with a FT-IR Spectrum 2000 (16 scans) spectrophotometer (Perkin Elmer, Waltham, MA, USA). ${ }^{1} \mathrm{H}-\mathrm{NMR}$ (Nuclear magnetic resonance) spectra were obtained using an Avance 500 (Bruker, Karlsruhe, Germany) at $500 \mathrm{MHz}$ for solutions in DMSO- $d_{6}$. Elemental analyses were carried out on a CHNS-932 (LECO Corporation, St. Joseph, MI, USA) for C, H, N, and S. The melting point was measured using an M-560 melting point instrument (Buchi, Flawil, Switzerland). The absorption spectra were measured in $1 \mathrm{~cm}$ quartz cells on an HP8453 UV-visible spectrophotometer (Agilent, Santa Clara, CA, USA). The reflectance and color properties (CIE L*, $\mathrm{a}^{*}, \mathrm{~b}^{*}, \mathrm{C}^{*}$, and $\mathrm{h}_{a b}$ ) of the dyed samples were measured using an X-Rite 8000 Series (X-Rite, Inc., Grand Rapids, MI, USA) in specific color measurement conditions (standard light D65, $10^{\circ}$ standard observer, and specular component included).

\subsection{Dye Synthesis and Preparation of Dye Dispersions}

Syntheses of $N$-methylphthalimidylazo dyes (P-dyes, P1-P6) (Scheme 3) and 4-nitroaminoazobenzene dyes (N-dyes, N1-N6) were carried out as described in the previous studies (Table 1) [12,13]. In total, $0.02 \mathrm{~mol}$ of diazo component was diazotized in $6.9 \mathrm{~mL}$ of $35 \% \mathrm{HCl}$ and $72 \mathrm{~mL}$ of water by adding $0.02 \mathrm{~mol}$ of $\mathrm{NaNO}_{2}$ at a temperature of $0-5^{\circ} \mathrm{C}$. After $5-6 \mathrm{~h}$, the solution of diazonium salt was added to a solution containing $0.02 \mathrm{~mol}$ of coupling component, $40 \mathrm{~mL}$ of water, and $3.4 \mathrm{~mL}$ of $35 \% \mathrm{HCl}$. After $5-6 \mathrm{~h}$, the precipitated dye was filtered, washed with water, and dried. The dyes were purified by crystallization from methanol. The structural analysis and characterization data of the synthesized dyes are given below.<smiles>[R][R]([R])c1ccc(N=Nc2ccc3c(c2)C(=O)N(C)C3=O)c([R])c1</smiles>

Scheme 3. Synthesis of azo disperse dye containing an $N$-methylphthalimide group.

Table 1. The synthesized dyes used in the present study.

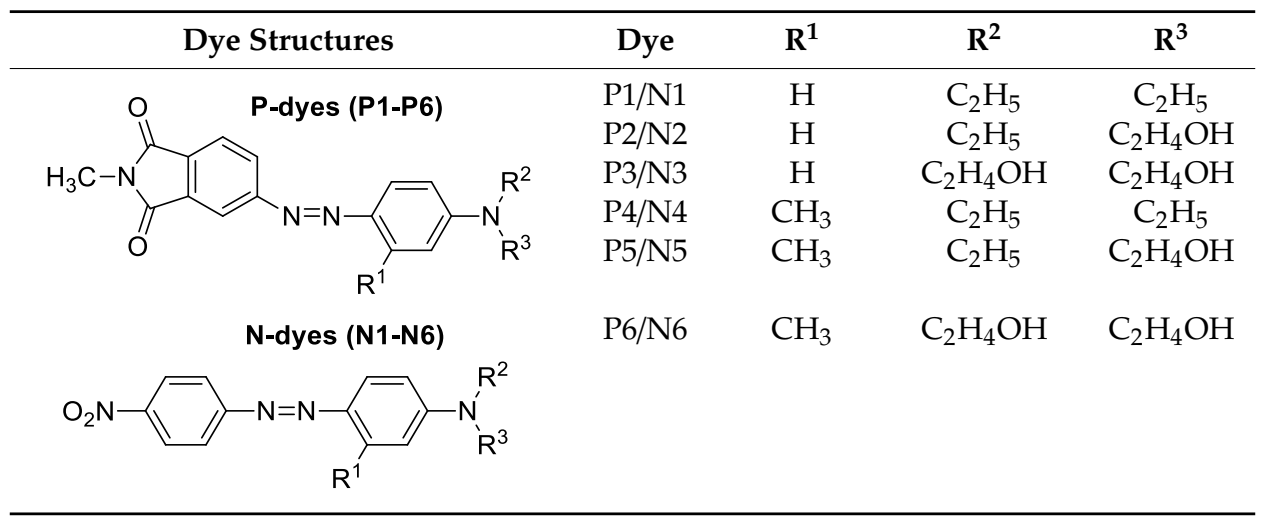


P1. (5-((4-(diethylamino)phenyl)diazenyl)-2-methylisoindoline-1,3-dione). Reddish orange solid. Yield 75.2\%. M.p. $187-188^{\circ} \mathrm{C}$. IR (KBr) v max: 2978 (w, CH), $1715(\mathrm{~s}, \mathrm{C}=\mathrm{O}), 1600,1518(\mathrm{~s}, \mathrm{ArC}=\mathrm{C})$, $1557(\mathrm{w}, \mathrm{N}=\mathrm{N}) \mathrm{cm}^{-1}$. ${ }^{1} \mathrm{H}$ NMR $\left(500 \mathrm{MHz}, \mathrm{DMSO}-\mathrm{d}_{6}\right) \delta: 1.17\left(6 \mathrm{H}, \mathrm{t}, J=7.2, \mathrm{~N}\left(\mathrm{CH}_{2} \mathrm{CH}_{3}\right)_{2}\right), 3.06$ $\left(3 \mathrm{H}, \mathrm{s}\right.$, pthalimide- $\left.\mathrm{CH}_{3}\right), 3.50\left(4 \mathrm{H}, \mathrm{q}, J=7.0, \mathrm{~N}\left(\mathrm{CH}_{2} \mathrm{CH}_{3}\right)_{2}\right), 6.84(2 \mathrm{H}, \mathrm{d}, J=9.5, \mathrm{ArH}), 7.84(2 \mathrm{H}, \mathrm{d}$, $J=9.5, \mathrm{ArH}), 7.93(1 \mathrm{H}, \mathrm{d}, J=9.5$, phthalimide- $\mathrm{H}), 8.00(1 \mathrm{H}, \mathrm{s}$, phthalimide- $\mathrm{H}), 8.12(1 \mathrm{H}, \mathrm{d}, J=8.0$, phthalimide-H) ppm. Anal. calcd for $\mathrm{C}_{19} \mathrm{H}_{20} \mathrm{~N}_{4} \mathrm{O}_{2}$ (\%): C 67.84; $\mathrm{H}$ 5.99; N 16.66, found: C 67.69; H 5.95; N 16.61.

P2. (5-((4-(ethyl(2-hydroxyethyl)amino)phenyl)diazenyl)-2-methylisoindoline-1,3-dione). Reddish orange solid. Yield 62.3\%. M.p. 196-197 ${ }^{\circ} \mathrm{C}$. IR (KBr) $v_{\max }: 3239$ (s, OH), 2978 (w, CH), 1714 (s, C = O), 1600, 1518 (s, ArC = C), 1555 (w, N = N), $1080(\mathrm{~m}, \mathrm{C}-\mathrm{O}) \mathrm{cm}^{-1} .{ }^{1} \mathrm{H}$ NMR $(500 \mathrm{MHz}$, DMSO-d $\left.{ }_{6}\right) \delta: 1.16\left(3 \mathrm{H}, \mathrm{t}, J=7.0, \mathrm{NCH}_{2} \mathrm{CH}_{3}\right), 3.05\left(3 \mathrm{H}, \mathrm{s}\right.$, phthalimide- $\left.\mathrm{CH}_{3}\right), 3.50-3.56(4 \mathrm{H}, \mathrm{m}$, $\left.\mathrm{NCH}_{2}\right), 3.631\left(2 \mathrm{H}, \mathrm{t}, J=5.9, \mathrm{NCH}_{2} \mathrm{CH}_{2} \mathrm{OH}\right), 4.84\left(1 \mathrm{H}, \mathrm{s}, \mathrm{NCH}_{2} \mathrm{CH}_{2} \mathrm{OH}\right), 6.87(2 \mathrm{H}, \mathrm{d}, J=9.0, \mathrm{ArH})$, $7.82(2 \mathrm{H}, \mathrm{d}, J=9.0, \mathrm{ArH}), 7.93(1 \mathrm{H}, \mathrm{d}, J=8.0$, phthalimide-H), $8.00(1 \mathrm{H}, \mathrm{s}$, phthalimide-H), 8.11 $\left(1 \mathrm{H}, \mathrm{d}, J=8.0\right.$, phthalimide-H) ppm. Anal. calcd for $\mathrm{C}_{19} \mathrm{H}_{20} \mathrm{~N}_{4} \mathrm{O}_{3}(\%)$ : C 64.76; $\mathrm{H}$ 5.72; N 15.90, found: C 64.58; H 5.69; N 15.86.

P3. (5-((4-(bis(2-hydroxyethyl)amino)phenyl)diazenyl)-2-methylisoindoline-1,3-dione). Reddish orange solid. Yield 59.4\%. M.p. $207-208{ }^{\circ} \mathrm{C}$. IR (KBr) v max: $3239(\mathrm{~s}, \mathrm{OH}), 2978(\mathrm{w}, \mathrm{CH})$, $1709(\mathrm{~s}, \mathrm{C}=\mathrm{O}), 1600,1518(\mathrm{~s}, \mathrm{ArC}=\mathrm{C}), 1560(\mathrm{w}, \mathrm{N}=\mathrm{N}), 1042(\mathrm{~m}, \mathrm{C}-\mathrm{O}) \mathrm{cm}^{-1} .{ }^{1} \mathrm{H}$ NMR $\left(500 \mathrm{MHz}, \mathrm{DMSO}_{6}\right)$ \&: $3.06\left(3 \mathrm{H}, \mathrm{s}\right.$, phthalimide- $\left.\mathrm{CH}_{3}\right), 3.59-3.63\left(8 \mathrm{H}, \mathrm{m}, \mathrm{NCH}_{2} \mathrm{CH}_{2}\right), 4.86(2 \mathrm{H}$, $\left.\mathrm{s}, \mathrm{N}\left(\mathrm{CH}_{2} \mathrm{CH}_{2} \mathrm{OH}\right)_{2}\right), 6.90(2 \mathrm{H}, \mathrm{d}, J=9.3, \mathrm{ArH}), 7.83(2 \mathrm{H}, \mathrm{d}, J=9.1, \mathrm{ArH}), 7.93(1 \mathrm{H}, \mathrm{d}, J=8.0$, phthalimide- $\mathrm{H}), 8.00(1 \mathrm{H}, \mathrm{s}$, phthalimide- $\mathrm{H}), 8.11(1 \mathrm{H}, \mathrm{d}, J=8.0$, phthalimide-H). Anal. calcd for $\mathrm{C}_{19} \mathrm{H}_{20} \mathrm{~N}_{4} \mathrm{O}_{4}$ (\%): C 61.95; $\mathrm{H}$ 5.47; N 15.21, found: C 61.78; H 5.42; N 15.17.

P4. (5-((4-(diethylamino)-2-methylphenyl)diazenyl)-2-methylisoindoline-1,3-dione). Red powder. Yield 81.0\%. M.p. $204-205^{\circ} \mathrm{C}$. IR (KBr) vmax: 2972 (w, CH), $1714(\mathrm{~s}, \mathrm{C}=\mathrm{O}), 1600,1511(\mathrm{~s}, \mathrm{ArC}=\mathrm{C})$, $1559(\mathrm{w}, \mathrm{N}=\mathrm{N}) \mathrm{cm}^{-1} .{ }^{1} \mathrm{H}$ NMR $\left(500 \mathrm{MHz}, \mathrm{DMSO}-\mathrm{d}_{6}\right) \delta: 1.18\left(6 \mathrm{H}, \mathrm{t}, J=7.0, \mathrm{~N}\left(\mathrm{CH}_{2} \mathrm{CH}_{3}\right)_{2}\right), 2.65$ $\left(3 \mathrm{H}, \mathrm{s}, \mathrm{ArCH}_{3}\right), 3.06\left(3 \mathrm{H}, \mathrm{s}\right.$, phthalimide- $\left.\mathrm{CH}_{3}\right), 3.49\left(4 \mathrm{H}, \mathrm{q}, J 7.2, \mathrm{~N}\left(\mathrm{CH}_{2} \mathrm{CH}_{3}\right)_{2}\right), 6.68(2 \mathrm{H}, \mathrm{d}, J=7.3$, $\mathrm{ArH}), 7.74(2 \mathrm{H}, \mathrm{d}, J=9.9, \mathrm{ArH}), 7.97(1 \mathrm{H}, \mathrm{d}, J=7.9$, phthalimide-H), $8.01(1 \mathrm{H}, \mathrm{s}$, phthalimide-H), $8.10\left(1 \mathrm{H}, \mathrm{d}, J=8.0\right.$, phthalimide-H). Anal. calcd for $\mathrm{C}_{20} \mathrm{H}_{22} \mathrm{~N}_{4} \mathrm{O}_{2}(\%): \mathrm{C} 68.55 ; \mathrm{H} 6.33 ; \mathrm{N} 15.99$, found: C 68.40; H 6.30; N 15.92.

P5. (5-((4-(ethyl(2-hydroxyethyl)amino)-2-methylphenyl)diazenyl)-2-methylisoindoline-1,3-dione). Red powder. Yield 67.2\%. M.p. $210-211^{\circ} \mathrm{C}$. IR (KBr) $v_{\max }: 3200$ (s, OH), 2978 (w, CH), 1714 $(\mathrm{s}, \mathrm{C}=\mathrm{O}), 1600,1505(\mathrm{~s}, \mathrm{ArC}=\mathrm{C}), 1557(\mathrm{w}, \mathrm{N}=\mathrm{N}), 1032(\mathrm{me}, \mathrm{C}-\mathrm{O}) \mathrm{cm}^{-1}$. ${ }^{1} \mathrm{H}$ NMR $(500 \mathrm{MHz}$, DMSO-d 6 ) $\delta: 1.16\left(3 \mathrm{H}, \mathrm{t}, J=7.0, \mathrm{NCH}_{2} \mathrm{CH}_{3}\right), 2.63\left(3 \mathrm{H}, \mathrm{s}, \mathrm{ArCH}_{3}\right) 3.05\left(3 \mathrm{H}, \mathrm{s}\right.$, phthalimide- $\left.\mathrm{CH}_{3}\right)$, 3.49-3.54 $\left(4 \mathrm{H}, \mathrm{m}, \mathrm{NCH}_{2}\right), 3.61\left(2 \mathrm{H}, \mathrm{t}, J=6.0, \mathrm{NCH}_{2} \mathrm{CH}_{2}-\mathrm{OH}\right), 4.83\left(1 \mathrm{H}, \mathrm{s}, \mathrm{NCH}_{2} \mathrm{CH}_{2} \mathrm{OH}\right)$, $6.69(2 \mathrm{H}, \mathrm{m}, \mathrm{ArH}), 7.70(1 \mathrm{H}, \mathrm{d}, J=9.9, \mathrm{ArH}), 7.94(1 \mathrm{H}, \mathrm{d}, J=7.8$, phthalimide- $\mathrm{H}), 7.97(1 \mathrm{H}, \mathrm{s}$, phthalimide-H), $8.07(1 \mathrm{H}, \mathrm{d}, J=7.9$, phthalimide- $\mathrm{H})$. Anal. calcd for $\mathrm{C}_{20} \mathrm{H}_{22} \mathrm{~N}_{4} \mathrm{O}_{3}(\%)$ : C 65.56; $\mathrm{H}$ 6.05; N 15.29, found: C 65.38; H 6.01; N 15.22.

P6. (5-((4-(bis(2-hydroxyethyl)amino)-2-methylphenyl)diazenyl)-2-methylisoindoline-1,3-dion). Reddish orange solid. Yield 62.3\%. M.p. 221-222 ${ }^{\circ} \mathrm{C}$. IR (KBr) $v_{\max }: 3226(\mathrm{~s}, \mathrm{OH}), 2988$ (w, $\mathrm{CH}), 1714$ (s, C = O), 1600, 1508 (s, ArC = C), $1566(\mathrm{w}, \mathrm{N}=\mathrm{N}), 1073(\mathrm{~m}, \mathrm{C}-\mathrm{O}) \mathrm{cm}^{-1} .{ }^{1} \mathrm{H}$ NMR $\left(500 \mathrm{MHz}, \mathrm{DMSO}-\mathrm{d}_{6}\right) \delta: 2.63\left(3 \mathrm{H}, \mathrm{s}, \mathrm{ArCH}_{3}\right), 3.04\left(3 \mathrm{H}, \mathrm{s}\right.$, phthalimide- $\left.\mathrm{CH}_{3}\right), 3.56-3.61(8 \mathrm{H}, \mathrm{m}$, $\left.\mathrm{N}\left(\mathrm{CH}_{2} \mathrm{CH}_{2} \mathrm{OH}\right)_{2}\right), 4.85\left(2 \mathrm{H}, \mathrm{s}, \mathrm{N}\left(\mathrm{CH}_{2} \mathrm{CH}_{2} \mathrm{OH}\right)_{2}\right), 6.70(2 \mathrm{H}, \mathrm{d}, J=9.5, \mathrm{ArH}), 7.68(2 \mathrm{H}, \mathrm{d}, J=10.0$, ArH), $7.94(1 \mathrm{H}, \mathrm{d}, J=9.9$, phthalimide-H), $7.96(1 \mathrm{H}, \mathrm{s}$, phthalimide- $\mathrm{H}), 8.07(1 \mathrm{H}, \mathrm{d}, J=7.9$, phthalimide-H). Anal. calcd for $\mathrm{C}_{20} \mathrm{H}_{22} \mathrm{~N}_{4} \mathrm{O}_{4}(\%)$ : C 62.82; $\mathrm{H}$ 5.80; N 14.65, found: C 62.65; $\mathrm{H}$ 5.79; N 14.60 .

N1. (N,N-diethyl-4-((4-nitrophenyl)diazenyl)aniline). Brownish red solid. Yield 89.3\%. M.p. 150-151 ${ }^{\circ} \mathrm{C}$. IR (KBr) v $v_{\max }: 2950(\mathrm{w}, \mathrm{CH})$, 1600, $1508(\mathrm{~s}, \operatorname{ArC}=\mathrm{C}), 1556(\mathrm{w}, \mathrm{N}=\mathrm{N}), 1512$, $1353\left(\mathrm{~s}, \mathrm{NO}_{2}\right) \mathrm{cm}^{-1} .{ }^{1} \mathrm{H}$ NMR (500 MHz, DMSO-d 6 ) $\delta: 1.17\left(6 \mathrm{H}, \mathrm{t}, J=7.0, \mathrm{~N}\left(\mathrm{CH}_{2} \mathrm{CH}_{3}\right)_{2}\right), 3.50(4 \mathrm{H}$, $\left.\mathrm{q}, J=7.2, \mathrm{~N}\left(\mathrm{CH}_{2} \mathrm{CH}_{3}\right)_{2}\right), 6.85(2 \mathrm{H}, \mathrm{d}, J=9.0, \mathrm{ArH}), 7.84(2 \mathrm{H}, \mathrm{d}, J=9.5, \mathrm{ArH}), 7.92(2 \mathrm{H}, \mathrm{d}, J=9.0$, 
$\mathrm{ArH}), 8.35(2 \mathrm{H}, \mathrm{d}, J=9.0, \mathrm{ArH})$. Anal. calcd for $\mathrm{C}_{16} \mathrm{H}_{18} \mathrm{~N}_{4} \mathrm{O}_{2}$ (\%): C 64.41; $\mathrm{H} 6.08 ; \mathrm{N} 18.78$, found: C 64.28; H 6.05; N 18.72 .

N2. (2-(ethyl(4-((4-nitrophenyl)diazenyl)phenyl)amino)ethanol): dark brownish red solid; yield $66.2 \%$.

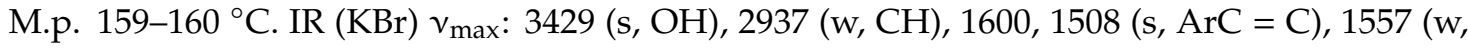
$\mathrm{N}=\mathrm{N}), 1512,1353\left(\mathrm{~s}, \mathrm{NO}_{2}\right), 1000(\mathrm{~m}, \mathrm{C}-\mathrm{O}) \mathrm{cm}^{-1} .{ }^{1} \mathrm{H}$ NMR $\left(500 \mathrm{MHz}, \mathrm{DMSO}-\mathrm{d}_{6}\right) \delta: 1.17(3 \mathrm{H}$, $\left.\mathrm{t}, J=7.0, \mathrm{NCH}_{2} \mathrm{CH}_{3}\right), 3.51-3.55\left(4 \mathrm{H}, \mathrm{m}, \mathrm{NCH}_{2}\right), 3.63\left(2 \mathrm{H}, \mathrm{t}, J=6.0, \mathrm{NCH}_{2} \mathrm{CH}_{2} \mathrm{OH}\right), 4.86(1 \mathrm{H}, \mathrm{s}$, $\left.\mathrm{NCH}_{2} \mathrm{CH}_{2} \mathrm{OH}\right), 6.87(2 \mathrm{H}, \mathrm{d}, J=9.0, \mathrm{ArH}), 7.82(2 \mathrm{H}, \mathrm{d}, J=9.0, \mathrm{ArH}), 7.95(2 \mathrm{H}, \mathrm{d}, J=8.0, \mathrm{ArH}), 8.10$ $(2 \mathrm{H}, \mathrm{d}, J=8.0, \mathrm{ArH})$. Anal. calcd for $\mathrm{C}_{16} \mathrm{H}_{18} \mathrm{~N}_{4} \mathrm{O}_{3}(\%)$ : C 61.13; $\mathrm{H} 5.77 ; \mathrm{N} 17.82$, found: $\mathrm{C} 60.94 ; \mathrm{H}$ 5.72; N 17.76 .

N3. (2,2'-((4-((4-nitrophenyl)diazenyl)phenyl)azanediyl)diethanol. Brownish red solid. Yield $60.8 \%$. M.p. $170-171^{\circ} \mathrm{C}$. IR (KBr) v $v_{\max }: 3390$ (s, OH), 2975 (w, CH), 1600, 1508 (s, $\left.\mathrm{ArC}=\mathrm{C}\right), 1559$ (w, $\mathrm{N}=\mathrm{N}), 1512,1333\left(\mathrm{~s}, \mathrm{NO}_{2}\right), 1048(\mathrm{~m}, \mathrm{C}-\mathrm{O}) \mathrm{cm}^{-1} .{ }^{1} \mathrm{H}$ NMR $\left(500 \mathrm{MHz}, \mathrm{DMSO}-\mathrm{d}_{6}\right) \delta: 3.62-3.67(8 \mathrm{H}$, $\left.\mathrm{m}, \mathrm{N}\left(\mathrm{CH}_{2} \mathrm{CH}_{2} \mathrm{OH}\right)_{2}\right), 4.89\left(1 \mathrm{H}, \mathrm{s}, \mathrm{N}\left(\mathrm{CH}_{2} \mathrm{CH}_{2} \mathrm{OH}\right)_{2}\right), 6.91(2 \mathrm{H}, \mathrm{d}, J=9.0, \mathrm{ArH}), 7.82(2 \mathrm{H}, \mathrm{d}, J=9.0$, $\mathrm{ArH}), 7.91(2 \mathrm{H}, \mathrm{d}, J=9.0, \mathrm{ArH}), 8.34(2 \mathrm{H}, \mathrm{d}, J=9.0, \mathrm{ArH})$. Anal. calcd for $\mathrm{C}_{16} \mathrm{H}_{18} \mathrm{~N}_{4} \mathrm{O}_{4}(\%)$ : C 58.17; H 5.49; N 16.96, found: C 58.00; H 5.42; N 16.90.

N4. (N,N-diethyl-3-methyl-4-((4-nitrophenyl)diazenyl)aniline). Violet solid. Yield 64.4\%. M.p. 164-165 ${ }^{\circ} \mathrm{C}$. IR (KBr) v $v_{\max }: 2928(\mathrm{w}, \mathrm{CH}), 1600,1508(\mathrm{~s}, \mathrm{ArC}=\mathrm{C}), 1559(\mathrm{w}, \mathrm{N}=\mathrm{N}), 1512,1359$ $\left(\mathrm{s}, \mathrm{NO}_{2}\right) \mathrm{cm}^{-1} .{ }^{1} \mathrm{H}$ NMR $\left(500 \mathrm{MHz}, \mathrm{DMSO}-\mathrm{d}_{6}\right) \delta: 1.16\left(6 \mathrm{H}, \mathrm{t}, J=7.0, \mathrm{~N}\left(\mathrm{CH}_{2} \mathrm{CH}_{3}\right)_{2}\right), 2.65(3 \mathrm{H}, \mathrm{s}$, $\left.\mathrm{ArCH}_{3}\right), 3.49\left(4 \mathrm{H}, \mathrm{q}, J=7.2, \mathrm{~N}\left(\mathrm{CH}_{2} \mathrm{CH}_{3}\right)_{2}\right), 6.85(2 \mathrm{H}, \mathrm{d}, J=9.0, \mathrm{ArH}), 7.74(1 \mathrm{H}, \mathrm{d}, J=10.0, \mathrm{ArH})$, $7.97(2 \mathrm{H}, \mathrm{d}, J=9.9, \mathrm{ArH}), 8.10(2 \mathrm{H}, \mathrm{d}, J=8.0, \mathrm{ArH})$. Anal. calcd for $\mathrm{C}_{17} \mathrm{H}_{20} \mathrm{~N}_{4} \mathrm{O}_{2}(\%): \mathrm{C} 65.37 ; \mathrm{H}$ 6.45; N 17.94, found: C 65.11; H 6.41; N 17.89.

N5. (2-(ethyl(3-methyl-4-((4-nitrophenyl)diazenyl)phenyl)amino)ethanol). Dark brownish red solid. Yield 58.9\%. M.p. 173-174 ${ }^{\circ} \mathrm{C}$. IR (KBr) $v_{\max }: 3271$ (s, OH), 2975 (w, CH), 1603, 1508 (s, ArC = C), $1559(\mathrm{w}, \mathrm{N}=\mathrm{N}), 1512,1355\left(\mathrm{~s}, \mathrm{NO}_{2}\right), 1044(\mathrm{~m}, \mathrm{C}-\mathrm{O}) \mathrm{cm}^{-1} .{ }^{1} \mathrm{H}$ NMR $\left(500 \mathrm{MHz}, \mathrm{DMSO}-\mathrm{d}_{6}\right) \delta$ : $1.16\left(3 \mathrm{H}, \mathrm{t}, J=7.0, \mathrm{NCH}_{2} \mathrm{CH}_{3}\right), 2.63\left(3 \mathrm{H}, \mathrm{s}, \mathrm{ArCH}_{3}\right) 3.50-3.54\left(4 \mathrm{H}, \mathrm{m}, \mathrm{NCH}_{2}\right), 3.62(2 \mathrm{H}, \mathrm{t}, J=6.0$, $\left.\mathrm{NCH}_{2} \mathrm{CH}_{2} \mathrm{OH}\right), 4.86\left(1 \mathrm{H}, \mathrm{s}, \mathrm{NCH}_{2} \mathrm{CH}_{2} \mathrm{OH}\right), 6.69(2 \mathrm{H}, \mathrm{d}, J=4.5, \mathrm{ArH}), 7.70(1 \mathrm{H}, \mathrm{d}, J=10.0, \mathrm{ArH})$, $7.94(2 \mathrm{H}, \mathrm{d}, J=8.0, \mathrm{ArH}), 8.08(2 \mathrm{H}, \mathrm{d}, J=8.0, \mathrm{ArH})$. Anal. calcd for $\mathrm{C}_{17} \mathrm{H}_{20} \mathrm{~N}_{4} \mathrm{O}_{3}(\%): \mathrm{C} 62.18 ; \mathrm{H}$ 6.14; N 17.06, found: C 62.01; H 6.11; N 17.01 .

N6. (2,2'-((3-methyl-4-((4-nitrophenyl)diazenyl)phenyl)azanediyl)diethanol). Dark brownish red solid. Yield 59.1\%. M.p. 184-185 ${ }^{\circ} \mathrm{C}$. IR (KBr) $v_{\max }: 3276$ (s, OH), 2930 (w, CH), 1603, 1508 (s, $\mathrm{ArC}=\mathrm{C}), 1558(\mathrm{w}, \mathrm{N}=\mathrm{N}), 1512,1340\left(\mathrm{~s}, \mathrm{NO}_{2}\right), 1067(\mathrm{~m}, \mathrm{C}-\mathrm{O}) \mathrm{cm}^{-1} .{ }^{1} \mathrm{H}$ NMR $\left(500 \mathrm{MHz}, \mathrm{DMSO}-\mathrm{d}_{6}\right)$ ঠ: $2.63\left(3 \mathrm{H}, \mathrm{s}, \mathrm{ArCH}_{3}\right), 3.59-3.63\left(8 \mathrm{H}, \mathrm{m}, \mathrm{N}\left(\mathrm{CH}_{2} \mathrm{CH}_{2} \mathrm{OH}\right)_{2}\right), 4.85\left(2 \mathrm{H}, \mathrm{s}, \mathrm{N}\left(\mathrm{CH}_{2} \mathrm{CH}_{2} \mathrm{OH}\right)_{2}\right), 6.70$ $(2 \mathrm{H}, \mathrm{d}, J=9.5, \mathrm{ArH}), 7.68(1 \mathrm{H}, \mathrm{d}, J=10.0, \mathrm{ArH}), 7.93(2 \mathrm{H}, \mathrm{d}, J=8.0, \mathrm{ArH}), 8.072(2 \mathrm{H}, \mathrm{d}, J=8.0$, ArH). Anal. calcd for $\mathrm{C}_{17} \mathrm{H}_{20} \mathrm{~N}_{4} \mathrm{O}_{4}$ (\%): C 59.29; $\mathrm{H}$ 5.85; $\mathrm{N}$ 16.27, found: C 59.02; $\mathrm{H} 5.82 ; \mathrm{N} 16.21$.

Dye dispersion (1.0 $w / v \%$ ) was prepared in water. Dispersing agent (Diwatex, $40 \%$ on weight of dye), wetting agent (Sandozin NIE Liquid, 1 drop), and $1.0 \mathrm{~g}$ of dye were milled with glass beads for $24 \mathrm{~h}$ in $100 \mathrm{~mL}$ water buffered at $\mathrm{pH} 4.0-4.5$.

\subsection{Dyeing}

Cellulose diacetate fabrics were dyed in a laboratory dyeing machine (DL-6000; Daelim Starlet Co. Ltd., Gyeonggi-do, Korea) at a liquor ratio of 20:1. A $40 \mathrm{~mL}$ dyebath was prepared using a dispersing agent, formulated dye dispersion, and distilled water. The cellulose diacetate fabric $(2.0 \mathrm{~g})$ was dyed in the dyebath for $60 \mathrm{~min}$ at $85^{\circ} \mathrm{C}$ and washed with soaping agent $(1.0 \mathrm{~g} / \mathrm{L})$ for $15 \mathrm{~min}$ at $45^{\circ} \mathrm{C}$.

The build-up properties of the dyes on cellulose acetate were investigated by measuring the color strength of dyed fabrics at various dye concentrations. K/S values were calculated from the reflectance values according to Kubelka-Munk theory and the color strength $\left(f_{k}\right)$ was determined from the sum of the weighed $\mathrm{K} / \mathrm{S}$ values in the visible region, given by [14]: 


$$
f_{k}=\sum_{\lambda=400}^{700}(K / S)_{\lambda}\left(\bar{x}_{10, \lambda}+\bar{y}_{10, \lambda}+\bar{z}_{10, \lambda}\right)
$$

where $\bar{x}_{10, \lambda}, \bar{y}_{10, \lambda}$, and $\bar{z}_{10, \lambda}$ are the color matching functions for the $10^{\circ}$ standard observer at each wavelength (ISO 7724/1-1984).

\subsection{Color Fastness Test}

To evaluate the color fastness properties of the synthesized dyes on cellulose diacetate fabrics, the dyed fabric was subjected to three different types of aftertreatment i.e., soaping, a hot-rinse and no-treatment, and heat-set at $150{ }^{\circ} \mathrm{C}$ for $45 \mathrm{~s}$ in a laboratory tenter (Tex-dryer, Daelim Starlet Co. Ltd., Gyeonggi-do, Korea). 1/1 standard depth was used for the color fastness test. Wash fastness was determined using international standards (ISO 105-C06 D3S) [15]. Changes in shade and staining of adjacent multifiber (Multifiber DW, adjacent fabric, BS EN ISO 105-F10, SDC L Enterprises Limited, Holmfirth, UK) [15] were assessed using grey scales. A numerical grading was given on a scale of 1 to 5 , where a higher number indicated better fastness.

\section{Results}

\subsection{Spectral Properties of the Synthesized Dyes}

As shown in Table 2, N-dye series exhibited higher extinction coefficients and half-band width, and therefore gave greater tinctorial strength as compared to P-dye series. The UV-Visible spectra of the synthesized dyes in ethanol solution are shown in Figure 3. Regarding the spectral properties of the synthesized dyes, the color ranges of P-dye series and N-dye series were almost identical, both of which fall into the color gamut of orange to red $(477-499 \mathrm{~nm})$. However, the extinction coefficient $\left(\varepsilon_{\max }\right)$ and half band-width $\left(\Delta \lambda_{1 / 2}\right)$ were at different levels. Among the P-dye series, P2, P3, and P6 showed very similar extinction coefficients to their analogs in $\mathrm{N}$-dye series. Therefore, we can expect P2, P3, and P6 will exhibit a relatively reasonable level of build-up properties. The value of half band-width is a useful criterion for the evaluation of the hue brightness of dyes [16]. Dyes with low half band-width have bright hues, while those with high half bandwidth have dull hues. Comparatively, P-dye series has lower half band-width than $\mathrm{N}$-dye series; thus, they are expected to have higher chromaticity on the dyed cellulose diacetate.

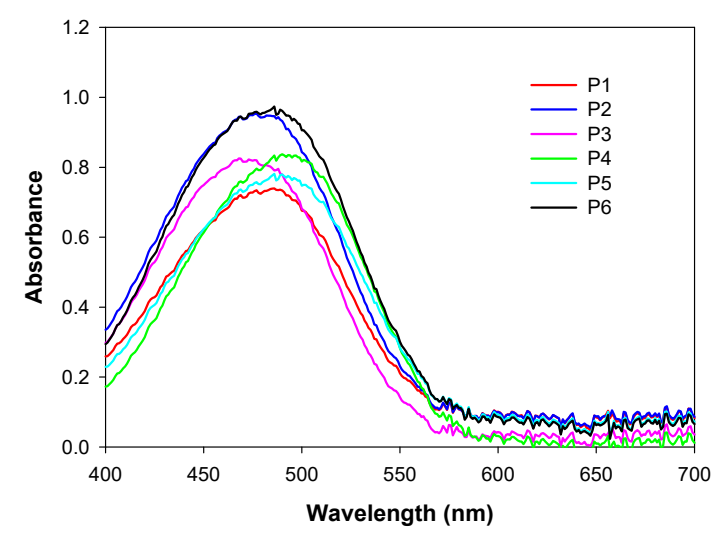

(a)

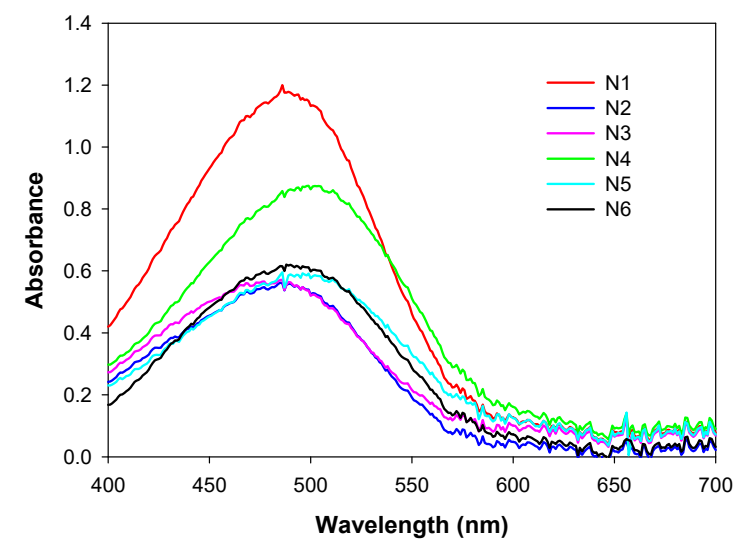

(b)

Figure 3. UV/Visible spectra of the synthesized dyes in ethanol: (a) P-dyes. (b) N-dyes. 
Table 2. The spectral properties of the synthesized dyes.

\begin{tabular}{|c|c|c|c|c|c|c|c|}
\hline Dye & $\begin{array}{c}\lambda_{\max }^{E t O H} \\
(\mathrm{~nm})\end{array}$ & $\begin{array}{c}\varepsilon_{\max }^{E t O H} \\
\left(\mathrm{Lmo}^{-1} \mathrm{~cm}^{-1}\right)\end{array}$ & $\begin{array}{c}\Delta \lambda_{1 / 2}^{E t O H} \\
(\mathrm{~nm})\end{array}$ & Dye & $\begin{array}{c}\lambda_{\max }^{E t O H} \\
(\mathrm{~nm})\end{array}$ & $\begin{array}{c}\varepsilon_{\max }^{\mathrm{EtOH}} \\
\left(\mathrm{Lmo}^{-1} \mathrm{~cm}^{-1}\right)\end{array}$ & $\begin{array}{c}\Delta \lambda_{1 / 2}^{E t O H} \\
(\mathbf{n m})\end{array}$ \\
\hline P1 & 485 & 21,400 & 115 & N1 & 486 & 35,800 & 125 \\
\hline P2 & 482 & 32,700 & 113 & N2 & 485 & 37,300 & 129 \\
\hline P3 & 477 & 34,400 & 109 & N3 & 486 & 35,800 & 134 \\
\hline P4 & 498 & 17,000 & 109 & N4 & 499 & 33,300 & 131 \\
\hline P5 & 486 & 29,800 & 118 & N5 & 486 & 33,500 & 138 \\
\hline P6 & 488 & 33,400 & 106 & N6 & 488 & 35,800 & 125 \\
\hline
\end{tabular}

\subsection{Dyeing Properties on Cellulose Diacetate}

\subsubsection{Buildup Properties on Cellulose Diacetate}

Figure 4 illustrates the relative color strength $\left(f_{k}\right)$ of dyed cellulose diacetate fabrics depending on the amount of dye applied (mass of fabric, \%omf). P-dyes exhibited slower buildup and reduced color yield at saturation points compared to $\mathrm{N}$-dyes. For example, the saturation points for P2 and P3 were between 1.0 and $2.0 \% \mathrm{omf}$, while those of $\mathrm{N} 2$ and N3 were between 2.0 and $4.0 \% \mathrm{omf}$. Thus, in dyeing cellulose diacetate fiber, the substantivity of $N$-methylphthalimidylazo dyes was shown to be lower than that of 4-aminoazobenzene dyes containing a $p$-nitro group. The relatively poor build-up properties of P-dye series can be explained by the relatively lower tinctorial strength and affinity to acetate due to the lower polarity. This explains why many commercial azo disperse dyes for PET (poly (ethylene terephthalate)) and acetate fibers contain the $p$-nitro group in the diazo component. Among the P-dye series, P3 showed the better build-up properties on cellulose diacetate compared to the other P-dyes probably because of its higher tinctorial strength and relatively lower hydrophobic character due to the inclusion of two hydrophobic hydroxy groups and no methyl group in its coupling component. In addition, the hydrogen bonding and polar interactions between hydroxyl groups of cellulose diacetate and the di(hydroxyethyl)amine moiety of the dye's structure could contribute to the better dyeability of P3.

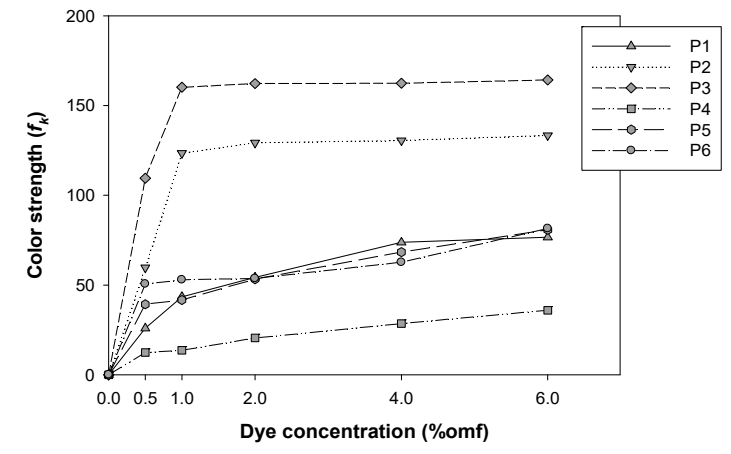

(a)

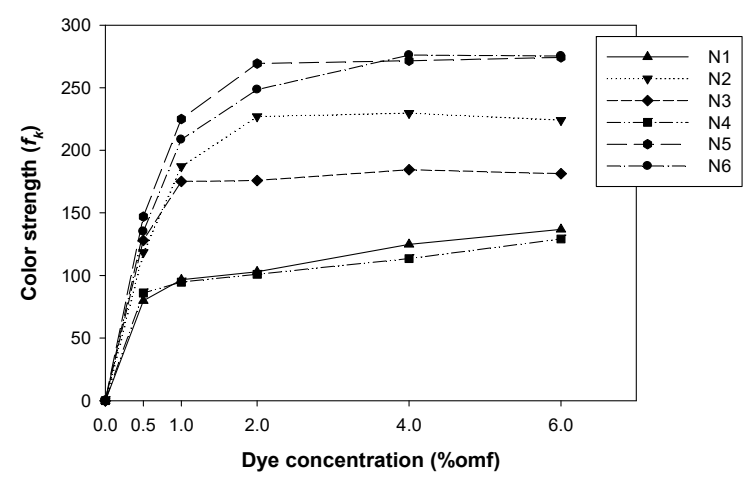

(b)

Figure 4. Build-up properties of the synthesized dyes on cellulose diacetate: (a) P-dyes. (b) N-dyes.

From the scatter plots showing the relationship between dyeability and hydrophobicity of dyes (Figure 5), it is clear that dyes with lower hydrophobicity exhibit better build-up properties on cellulose diacetate fibers. This is due to the relatively lower hydrophobic nature of cellulose acetate fibers compared to that of PET. P1, P4, N1, and N4 with the higher $\log \mathrm{P}$ values $(\mathrm{P} 1=4.39, \mathrm{P} 4=4.87$, $\mathrm{N} 1=4.42, \mathrm{~N} 4=4.83$ ) due to the hydrophobic $N, N$-diethyl group showing the lower build-up property on cellulose diacetate fibers. Cellulose acetate is not too hydrophobic, nor is it hydrophilic. This results in moisture-retention properties that relate to the excellent comfort factor [17]. 


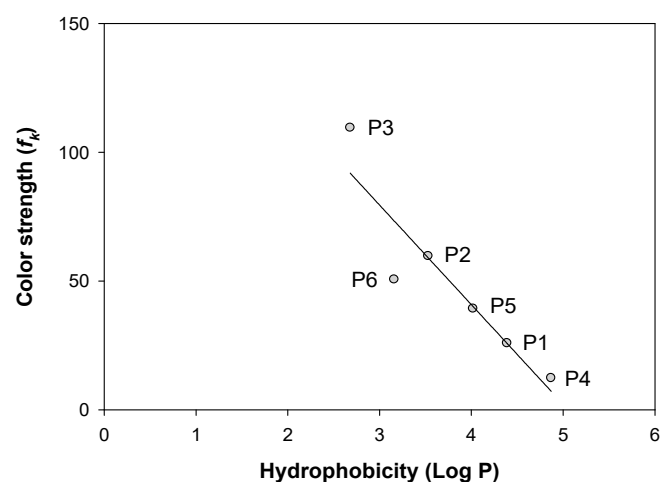

(a)

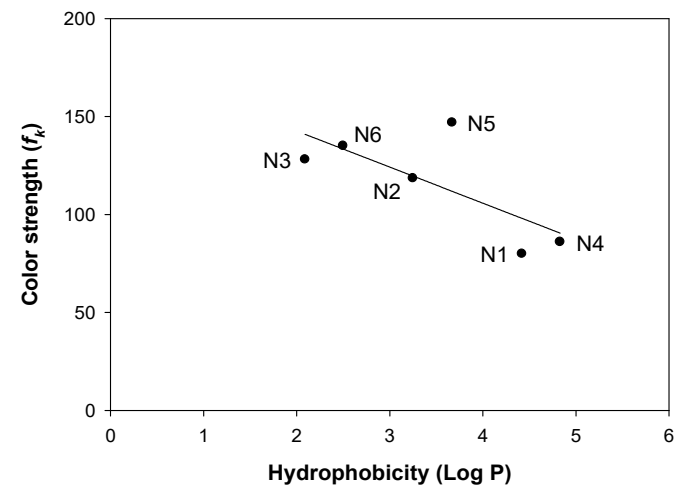

(b)

Figure 5. Correlation between hydrophobicity $(\log \mathrm{P})$ and color strength $\left(f_{k}\right)$ of the synthesized dyes on cellulose diacetate: (a) P-dyes. (b) N-dyes.

\subsubsection{Color Properties on Cellulose Diacetate}

The colorimetric properties of dyes, expressed in terms of the CIE (Commission International $\mathrm{d}^{\prime}$ Eclairage) $1976 \mathrm{~L}^{*} \mathrm{a}^{*} \mathrm{~b}^{*}$ color space parameters, as shown in Table 3, expresses the dyeing behavior of dyes. The colors of synthesized N-dyes and P-dyes on cellulose diacetate fall into the range of orange to red. P-dyes (P1-P6) show relatively higher chroma ( $\left.\mathrm{C}^{*}\right)$ than $\mathrm{N}$-dyes (N1-N6) (Figure 6). As expected, $\mathrm{P}$-dyes were slightly hypsochromic on cellulose diacetate compared with $\mathrm{N}$-dyes. This can be explained by the relatively lower electron-withdrawing power of the $N$-methylphthalimidyl group compared to 4-nitroanilne. These trends in colorimetric properties are consistent with their spectral properties in solvent, as shown in $\lambda_{\max }$ in Table 2 and $h_{a b}$ in Table 3 . Additionally, most P-dyes showed higher chromaticity values on the cellulose diacetate fibers compared to $\mathrm{N}$-dyes. This property can be explained by their narrower half-band width values shown in Table 2. Color photographs of the dyed cellulose diacetate fabrics are shown in Table 4.

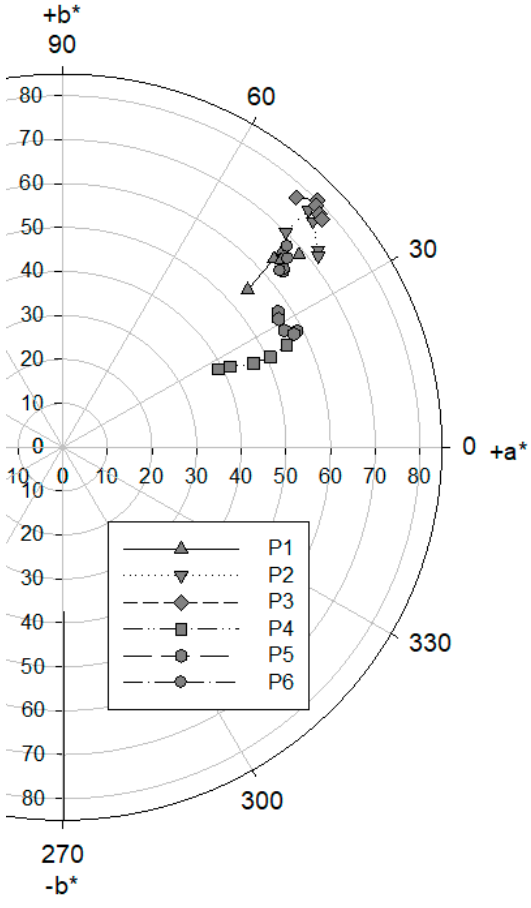

(a)

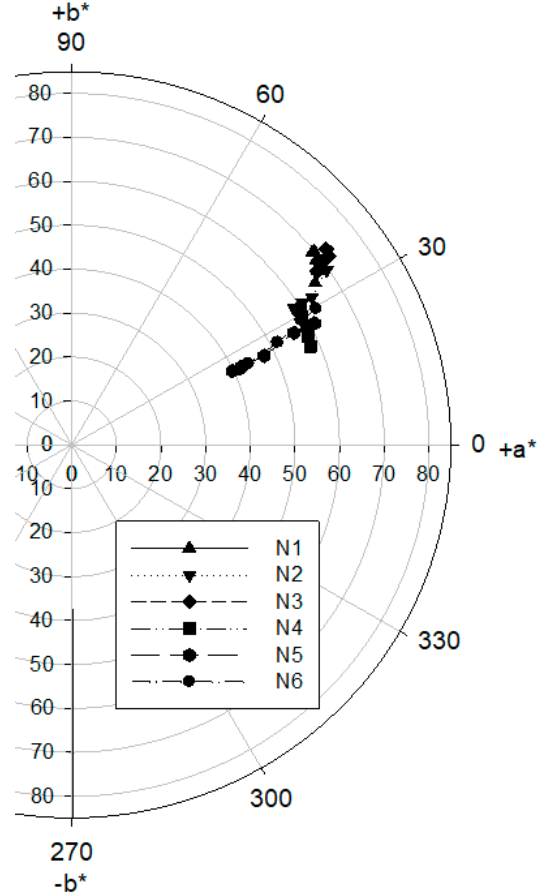

(b)

Figure 6. CIELAB color coordinates $\left(a^{*}\right.$ and $\left.b^{*}\right)$ of the cellulose diacetate fibers dyed with the synthesized dyes: (a) P-dyes. (b) N-dyes. 
Table 3. Colorimetric data and visual appearance of dyed cellulose diacetate fabrics with P-dyes and $\mathrm{N}$-dyes at $1.0 \%$ omf dye concentration.

\begin{tabular}{ccccccc}
\hline Dye & $\mathbf{L}^{*}$ & $\mathbf{a}^{*}$ & $\mathbf{b}^{*}$ & $\mathbf{C}^{*}$ & $\boldsymbol{h}_{\boldsymbol{a b}}$ & Color on Cellulose Acetate \\
\hline P1 & 62.9 & 47.3 & 42.9 & 63.8 & 42.2 & Strong reddish orange \\
P2 & 54.6 & 55.0 & 53.9 & 77.0 & 44.4 & Vivid reddish orange \\
P3 & 52.2 & 57.0 & 56.1 & 80.0 & 44.5 & Vivid reddish orange \\
P4 & 68.2 & 37.5 & 18.3 & 41.7 & 26.0 & Strong yellowish pink \\
P5 & 58.4 & 48.4 & 29.1 & 56.5 & 31.0 & Deep yellowish pink \\
P6 & 59.1 & 49.6 & 40.4 & 64.0 & 39.1 & Strong reddish orange \\
N1 & 48.9 & 55.0 & 39.5 & 67.7 & 34.0 & Vivid reddish orange \\
N2 & 42.4 & 57.0 & 39.7 & 69.5 & 34.8 & Vivid red \\
N3 & 45.0 & 57.5 & 42.9 & 71.7 & 36.8 & Vivid red \\
N4 & 43.2 & 53.0 & 24.5 & 58.4 & 24.8 & Strong red \\
N5 & 33.5 & 49.8 & 25.5 & 55.9 & 27.1 & Vivid red \\
N6 & 35.8 & 51.3 & 28.3 & 58.6 & 28.9 & Vivid red \\
\hline
\end{tabular}

Table 4. Color photographs of cellulose diacetate fabrics dyed with the synthesized dyes.

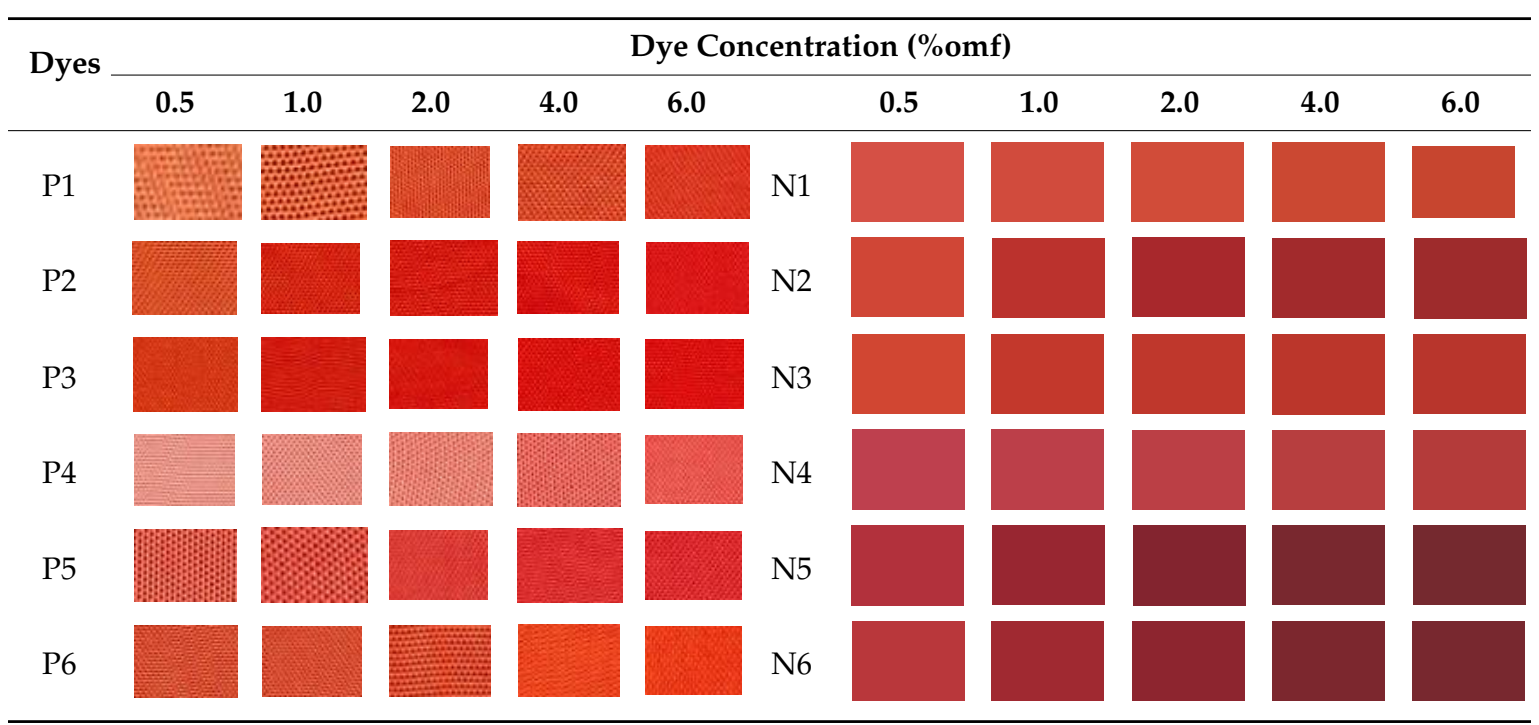

\subsection{Color Fastness to Washing}

Table 5 shows the wash fastness test result at $1 / 1$ standard depth dyeing with $\mathrm{N}$-dyes and P-dyes. For this test, P3 was chosen because the other P-dyes cannot reach the 1/1 standard depth even at the heaviest dyeing concentration due to their poor build-up properties. In order to compare their fastness properties, the analog of nitroaniline-based dyes N3 was chosen. The acetate and nylon components of adjacent multifiber fabrics are prone to staining during the wash fastness test of disperse-dyed cellulose diacetate fibers. In the case of the dyed cellulose diacetate fabrics being aftertreated with soaping, the staining on wool, acryl, PET, and cotton components of adjacent multifiber fabrics was in the range of 4-5 to 5 for P3 and 4 to $4-5$ for N3, respectively. Not only the aftertreated sample with soaping but also non-treated and hot rinsed samples after dyeing exhibited similarly excellent levels of wash fastness, presumably because the phthalimide group of the synthesized dyes was hydrolyzed to solubilizing diester groups during soaping aftertreatment, which involves weak alkaline with a $\mathrm{pH}$ of around $9-10$, as shown in previous studies $[10,11]$. The dye residues were, therefore, more readily removed during the wash-off and exhibited low staining on adjacent multifiber fabric in the wash fastness test. However, in the case of N3, even the aftertreated sample with soaping did not show an improved wash fastness level compared to non-treated and hot rinsed samples. An analogous comparison between P3 and N3 revealed a gap in wash-off property, exemplified by the staining of nylon (Figure 7). P3 exhibited higher wash fastness than N3 by as much as about 2.0 grading units, irrespective of the 
aftertreatment methods. The overall results show that $N$-methylphthalimidylazo dyes have excellent wash fastness on cellulose diacetate fibers compared to the conventional 4-nitro-4'-nitroazobenzene disperse dyes.

Table 5. Fastness to washing of dyed cellulose diacetate fabrics with dyes P3 and N3 (ISO 105 C06 D3S) at $1 / 1$ standard depth dyeing.

\begin{tabular}{ccccccccc}
\hline \multirow{2}{*}{ Dye } & \multirow{2}{*}{ After-Treatment } & \multicolumn{9}{c}{ Staining } & \multicolumn{2}{c}{ Change } \\
\cline { 3 - 8 } & Wool & Acryl & PET & Nylon & Cotton & Acetate & \\
\hline \multirow{3}{*}{ P3 } & No-treatment & $4-5$ & 5 & $4-5$ & $4-5$ & $4-5$ & 4 & $4-5$ \\
& Hot-rinse & 5 & 5 & $4-5$ & $4-5$ & $4-5$ & 4 & $4-5$ \\
& Soaping & 5 & 5 & 5 & $4-5$ & 5 & $4-5$ & $4-5$ \\
\hline \multirow{3}{*}{ N3 } & No-treatment & 4 & $4-5$ & $4-5$ & $2-3$ & 4 & $2-3$ & $2-3$ \\
& Hot-rinse & 4 & $4-5$ & $4-5$ & $2-3$ & 4 & $2-3$ & $2-3$ \\
& Soaping & $4-5$ & $4-5$ & $4-5$ & $2-3$ & $4-5$ & $2-3$ & $2-3$ \\
\hline
\end{tabular}

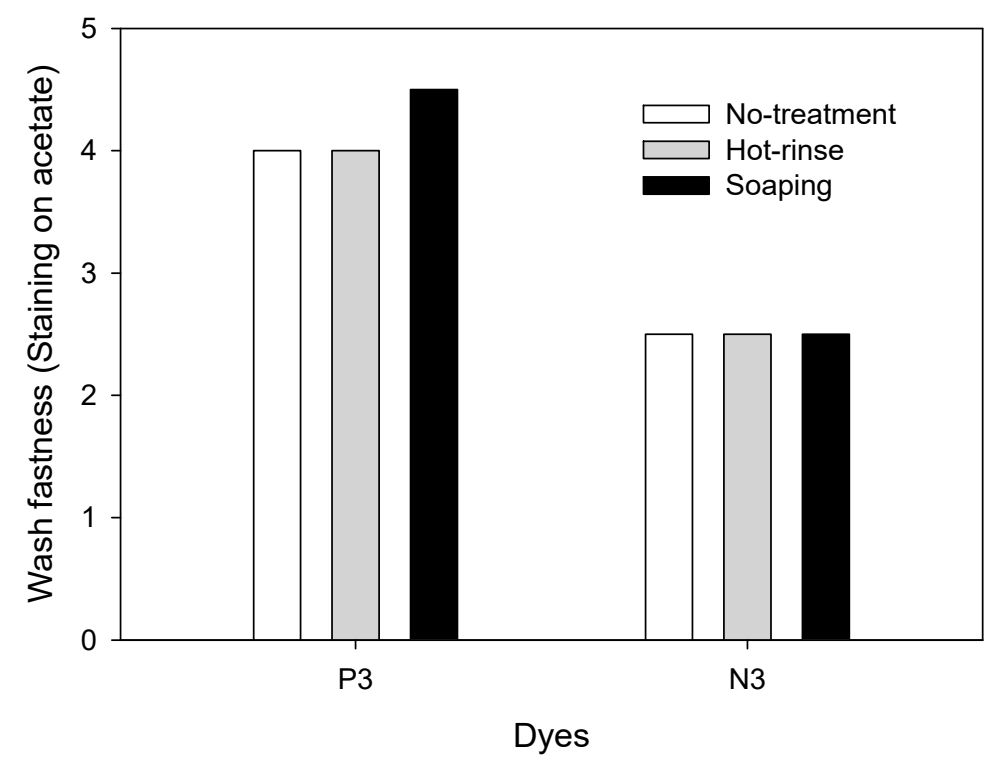

Figure 7. Wash fastness of cellulose diacetate fabrics dyed with dyes P3 and N3 at 1/1 standard depth dyeing.

\section{Conclusions}

Our present study investigated the dyeing and fastness properties of $N$-methylphthalimide-based azo disperse dyes on cellulose diacetate. $\mathrm{N}$-methylphthalimidylazo disperse dyes showed superior wash fastness properties as compared to conventional 4 -aminoazobenzene disperse dyes. These results are probably attributable to the excellent wash-off properties resulting from the easy clearability imparted by the ring opening and ionization of phthalimide ring structure. Excellent wash fastness was exhibited in soaping and non-treated and hot rinsed aftertreatment samples, presumably because the phthalimide groups of the synthesized dyes were hydrolyzed to solubilizing diester groups during aftertreatment. The dye residues were, therefore, more readily removed during the wash-off and exhibited low staining on adjacent multifiber fabric in the wash fastness test. These findings are consistent with the hydrolysis analysis results using HPLC and a UV-visible spectrophotometer in the previous studies [10].

$\mathrm{N}$-methylphthalimide-based azo disperse dyes showed relatively lower build-up properties probably because of their low tinctorial strength and affinity to acetate fibers. With regard to hydrophobicity/hydrophilicity balance, the dyes with low hydrophobicity exhibited better build-up 
properties on cellulose diacetate fibers. This is presumably as a result of the relatively less hydrophobic nature of cellulose acetate fibers as compared to that of PET.

Results of our present study demonstrate $N$-methylphthalimidylazo disperse dyes as a promising, efficient alternative to high value-added dyes that can be used for high color fastness dyeing of cellulose diacetate with a minimal discharge of wastewater if their build-up properties are improved to a satisfactory level.

Author Contributions: Conceptualization, S.Y.; data curation, S.Y.; formal analysis, H.K., E.L., and K.H.H. investigation, S.Y. and H.K.; methodology, E.L., N.O., and S.K.; supervision, J.K.; writing-original draft, K.H.H.; writing-review and editing, J.K. All authors have read and agreed to the published version of the manuscript.

Funding: This research was funded by the Korea Institute for Advancement of Technology (KIAT), the Ministry of Trade, Industry and Energy (MOTIE) (P0012770), the National Research Foundation of Korea (NRF), and the Ministry of Science and ICT (MSIT), Korea (2020R1A2C1006693).

Acknowledgments: This work was partly supported by the National Research Foundation of Korea (NRF) grant funded by the Korea government (MSIT) (2020R1A2C1006693) and the Korea Institute for Advancement of Technology (KIAT) grant funded by the Korea government (MOTIE) (P0012770).

Conflicts of Interest: The authors declare no conflict of interest.

\section{Abbreviations}

$(\mathrm{IR}) \mathrm{w}=$ weak; $\mathrm{m}=$ medium; $\mathrm{s}=$ strong $\left({ }^{1} \mathrm{H}\right.$ NMR $) \mathrm{s}=$ singlet; $\mathrm{d}=$ doublet; $\mathrm{q}=$ quartet; $\mathrm{m}=$ multiplet.

\section{References}

1. Abdul Khalil, H.P.S.; Bhat, I.U.H.; Jawaid, M.; Zaidon, A.; Hermawan, D.; Hadi, Y.S. Bamboo fibre reinforced biocomposites: A review. Mater. Des. 2012, 42, 353-368. [CrossRef]

2. $\quad$ Abdul Khalil, H.P.S.; Lai, T.K.; Tye, Y.Y.; Paridah, M.T.; Fazita, M.R.N.; Azniwati, A.A.; Dungani, R.; Rizal, S. Preparation and characterization of microcrystalline cellulose from sacred Bali bamboo as reinforcing filler in seaweed-based composite film. Fibers Polym. 2019, 19, 423-434. [CrossRef]

3. Chávez-Guerrero, L.; Esneider, M.; Bonilla, J.; Toxqui-Terán, A. Eco-friendly extraction of fibrils with hierarchical structure assisted by freeze-drying using Agave Salmiana leaves as a raw material. Fibers Polym. 2020, 21, 66-72. [CrossRef]

4. Hamed, O.A.; Jodeh, S.; Al-Hajj, N.; Hamed, E.M.; Abo-Obeid, A.; Fouad, Y. Cellulose acetate from biomass waste of olive industry. J. Wood Sci. 2015, 61, 45-52. [CrossRef]

5. Heinze, T.; Liebert, T. Chemical characteristics of cellulose acetate. In Cellulose Acetates: Properties and Applications; Rustemeyer, P., Ed.; Wiley: Weinham, Germany, 2004; pp. 167-238.

6. Rouette, H.K. Encyclopedia of Textile Finishing; Springer: Berlin/Heidelberg, Germany, 2000; pp. 255-257.

7. Broadbent, A.D. Basic Principles of Textile Coloration; SDC: Bradford, UK, 2001; pp. 11-14.

8. Koh, J. Dyeing with Disperse Dyes. In Textile Dyeing; Hauser, P., Ed.; Intech Open Limited: London, UK, 2011; pp. 207-209. ISBN 978-953-307-704-8.

9. Burkinshaw, S.M. Chemical Principles of Synthetic Fibre Dyeing; Chapman and Hall: Glasgow, UK, 1995; pp. 68-69.

10. Mamun Kabir, S.M.; Eom, M.; Lee, J.; Chae, D.E.; Baek, S.; Koh, J. Investigation of alkaline hydrolysis of phthalimide-based azo dye and its application to after-treatment optimization for high-fastness dyeing of polyesters. Color. Technol. 2018, 134, 206-213. [CrossRef]

11. Koh, J.S.; Kim, J.P. Synthesis of phthalimide-based alkali-dischargeable azo disperse dyes and analysis of their alkali-hydrolysis mechanism. Dyes Pigment. 1998, 37, 265-272. [CrossRef]

12. Koh, J.; Kim, H.; Park, J. Synthesis and spectral properties of phthalimide based alkali-clearable azo disperse dyes. Fibers Polym. 2009, 9, 143-151. [CrossRef]

13. Koh, J. Synthesis and Application of Alkali-Clearable Azo Disperse Dyes Containing a Fluorosulfonyl Group and Analysis of Their Alkali-Hydrolysis Kinetics. Ph.D. Thesis, Seoul National University, Seoul, South Korea, August 2002. 
14. Baumann, W.; Groebel, B.T.; Krayer, M.; Oesch, H.P.; Brossman, R.; Kleinmeier, N.; Leaver, A.T. Determination of relative colour strength and residual colour difference by means of reflectance measurements. J. Soc. Dye. Colour. 1987, 103, 100-105.

15. ISO 105-C06: 2010. Textiles_-Tests for Colour Fastness_Part C06: Colour Fastness to Domestic and Commercial Laundering; International Organization for Standardization: Geneva, Switzerland. Available online: https: //www.iso.org/obp/ui\#iso:std:iso:105:-C06:ed-4:v1:en (accessed on 31 October 2020).

16. Gordon, P.F.; Gregory, P. Organic Chemistry in Colour; Springer: New York, NY, USA, 1983; pp. $126-131$.

17. Lewin, M.; Pearce, E.M. Handbook of Fiber Chemistry, 2nd ed.; Marcel Dekker, Inc.: New York, NY, USA, 1998; pp. 834-835.

Publisher's Note: MDPI stays neutral with regard to jurisdictional claims in published maps and institutional affiliations.

(C) 2020 by the authors. Licensee MDPI, Basel, Switzerland. This article is an open access article distributed under the terms and conditions of the Creative Commons Attribution (CC BY) license (http://creativecommons.org/licenses/by/4.0/). 